\title{
A personalized Virtual Reality Experience for Relaxation Therapy
}

\author{
Joris Heyse, Thomas De Jonge, Maria Torres Vega, Femke De Backere and Filip De Turck \\ IDLab, Department of Information Technology, Ghent University - imec \\ E-mail: joris.heyse@ugent.be
}

\begin{abstract}
Virtual Reality (VR) has the potential to change not only to the way we consume and perceive entertainment but also to improve other important areas of society. One sector that is starting to benefit from the advantages of $V R$ is the treatment of stress related mental illnesses. VR is able to bring relaxation therapy to the next level in which solutions can be scalable (without the need for real-time dedicated professionals) and personalized. This paper presents VRelax, a personalized VR relaxation therapy approach. By means of semantic methodologies and online learning techniques, VRelax provides a personalized, relaxing virtual environment to the user.
\end{abstract}

Index Terms-Virtual Reality, Relaxation Therapy, Personalization

\section{INTRODUCTION}

The demand of virtual reality (VR) is booming thanks to the advancements in consumer electronics. This circumstance allows for the user to already nowadays benefit from fully immersive experiences at low cost. The multimedia and entertainment sector has been profiting from the advantages of VR for already some years, with the big Internet vendors such as YouTube and Facebook offering omnidirectional videos among their content. The technological advances in computation speed and graphics processing are giving a new impulse to the VR research to integrate it in other sectors with a higher societal and economical impact. One of the possible applications of VR is the healthcare sector, in particular on the treatment of mental health problems. There, VR can enable the assessment of cognitions, emotions and behavior in an ecologically valid environment [1].

Stress has been cited as the second most frequent health problem afflicting individuals' health and well-being [2]. It refers to the person's physiological responses to any demand or request perceived to be threatening to the physical, emotional or psychological health [3]. While at moderate levels, stress can be beneficial to the individuals, as it increases or becomes persistent it can contribute to poor mental health. Relaxation therapy has shown very promising results to cope or reduce the effects of extreme or long term stress. In the most traditional relaxation setup, therapist and patient sit in the same space, while the therapist uses a variety of methods (including progressive muscle relaxation, mental imaging, music, and even aromas) to induce a natural state of relaxation [4]. In other cases, stress patients turn to relaxation trainings such as yoga or meditation. These techniques, while effective, suffer from two issues. First, the need for a teaching environment, material and a dedicated therapist to lead the sessions, increases the costs and dramatically reduces the scalability of the approaches. Second, these techniques are designed for a generic patient (user). This means that adaptations to the different needs of the possible users are non-existant. VR relaxation therapy can potentially solve these two issues by offering personalized, low cost virtual experiences.

In the last years, several approaches showing the advantages of VR for improving mental health have appeared [1]. Shah et al. in [2] used VR therapy for stress management for people affected by mood disorder. They reported a decrease in stress, anxiety, and depression of the subjects. Gerber et al. [5] explored the effects of a visio-acoustic stimulation by means of VR for critically ill patients in the intensive care unit of a hospital. The VR stimulation had a significant impact on the relaxation of the patients as it was shown by the heart rate and blood pressure reductions. In [6], the authors explored the effects of immersive natural scenes presented with VR on people living or working in isolated and confined environments, confirming the benefits of the VR environments. Additionally, they argued about the need of personalized environments to improve the user's experience, and to enhance the effects of the relaxation therapy. Personalization is, indeed, one of the open questions in the literature of VR for relaxation therapy. As most of the approaches of the state-of-the-art provide the static environments to all the participants, the experience enhancement derived from a fully personalized environment is unexplored.

In this work we present VRelax, a personalized VR environment for relaxation therapy. By means of semantic methodologies such as ontologies and self-learning techniques, our method allows for the virtual environments to adapt to user preferences and real-time mood changes. The remainder of this paper presents first the working principles of VRelax. Then, the implementation details and showcase at the conference are discussed.

\section{VRELAX WORKING COMPONENTS}

The working principles of VRelax can be split into three major blocks (Figure 1). First, a model is needed to consolidate data from different sources. This model holds the needed personality data as well as real time stress data. It is used by personalization and adaptation algorithms to change the appearance of the VR environment, in order to match the requirements of the person. Finally, the decisions made by 


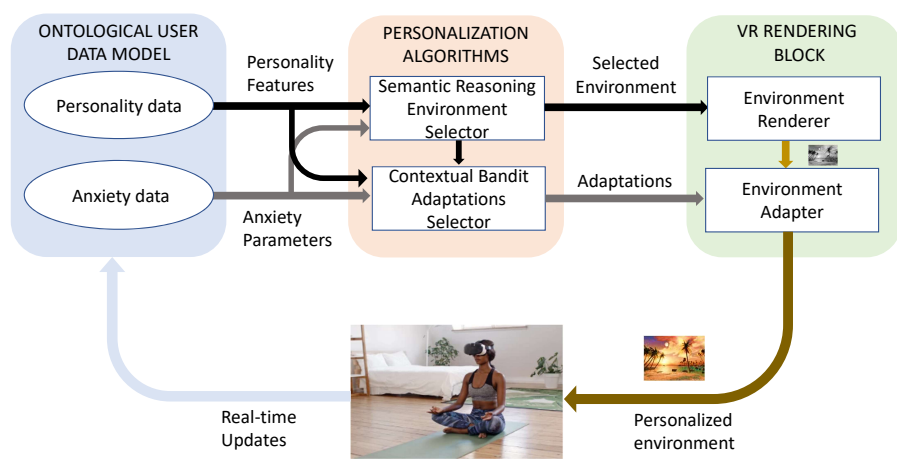

Fig. 1: Block diagram of the personalized Virtual Reality personalized relaxation system.

the algorithms are fed to the rendering component, which handles the real-time visualization of the personalized VR environment. The images produced by the rendering block are presented to the user through a Head Mounted Display (HMD). In response to seeing these renderings, real-time stress data can be sampled and fed to the user model. This effectively closes the feedback loop and allows for continues dynamic adaptations to the VR scene based on real time user data and background information. The innovations of VRelax are focussed on the user data and personalization blocks. The technological approaches for both are discussed in further detail in the next two Sections.

\section{A. User data model}

Combining all the relevant user data is not straightforward. In fact, while there are data, such as preferences and profile informations, which are static, others such as the real-time stress data, are dynamic, changes over time, and requires real time action. Semantic web technologies offer a solution for consolidating this data in an efficient manner, while allowing easy access to it. Additionally, semantic web technologies allow to reason about the data in order to extract high level knowledge from low level data [7]. A semantic ontology models concepts and relationships between those concepts in order to reason about data that conforms to that ontology. Therefore, while the highest layer of the ontology models generic concepts, the lower layers model more specific concepts and relations and provide a more fine-grained classification of concepts.

In this case, we use a semantic ontology to model, for a particular person, what is believed to provide relaxation to him/her. Linked to this assumption, there will be observations that either support or discard this believe. In fact, the ontology models the link between a stimulus and the response for a particular user. To build our ontology, we started from the layered ontology proposed in [8], which models patient characteristics in terms of fearful stimuli. While the original focus of this model was specifically to find stimuli that induce anxiety and stress, the higher level data model concepts that it uses, can be mapped into a relaxation therapy. In fact, stimuli and response relationships are modeled in the same way, where the stimuli and the interpretation of the response are application specific. Thus, in order to use the layered ontology from [8], application specific ontologies are needed for the low level layers which model the specific concepts in this application. This includes modeling the adaptable stimuli that are implemented in the VR environments and modeling the responses, these are the real-time measurements. In addition, application specific reasoning rules are defined, which allow for more easily extracting additional knowledge from the data model. Specifically, logical consequences of observing some response can be defined using such rules. For example, when an environment causes stress to a patient in a certain context, then it is not a suitable environment for relaxation purposes.

\section{B. Personalization algorithms}

The personalization component of our method consists of two individual algorithms. While, the first decides on the specific environment that will be shown, the second provides the specific real-time environment adaptations. Both algorithms take static personality data and dynamic anxiety data as input for making decisions. Two different approaches are envisioned for the implementation of these personalization algorithms. The environment decision relies on semantic reasoning and is used for the environment selection because the human preference does not change much over time. The second approach, i.e., the real-time adaptations, is performed by means of contextual multi-armed bandit learning [9], a subgroup of reinforcement learning.

The design of the algorithm for selecting a relaxation environment requires a lot of domain knowledge because it is based on manual formulation of rules that describe human behavior. Specifically, it formulates how certain responses affect the preferences of a user for a specific environment. The algorithm consists of applying a semantic reasoner on the data in the model and the manually defined rule. This reasoner evaluates these rules and expresses new knowledge, which dictate the decision of the algorithm. This enables an intelligent system that is solely based on knowledge from expert knowledge.

For the real-time adaptation algorithms, a self-learning approach is chosen. Specifically, contextual multi-armed bandits are the best suited option. These learning algorithms take some context, in this case personality data and anxiety data, as input and try to choose one of many possible actions, in this case an action is adapting the value of a parameter in the VR environment. Contextual bandit agents learn over time by receiving rewards based on their action. Specifically, in this case, the reward can be calculated from the physiological response. Positive decisions will result in reduced stress and increased comfort while negative decisions have the inverse effect.

\section{Experimental Demonstrator And ShowCASe}

VRelax has been implemented for deployment on the test bed shown in Figure 2. It is composed by a VR-enabled server connected to an HMD, in particular the HTC Vive ${ }^{1}$ due

\footnotetext{
${ }^{1}$ https://www.vive.com/eu/
} 


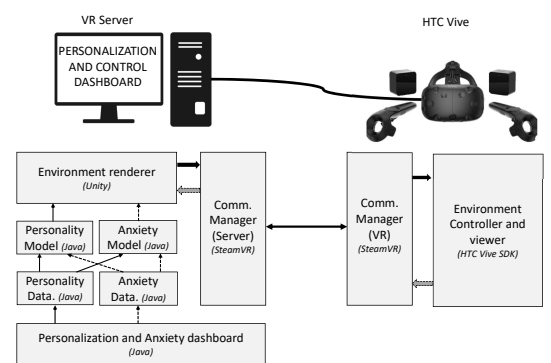

Fig. 2: Software and hardware implementation of VRelax.

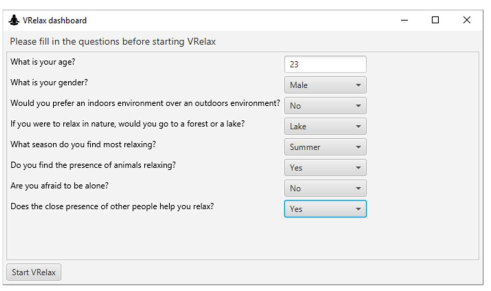

(a)

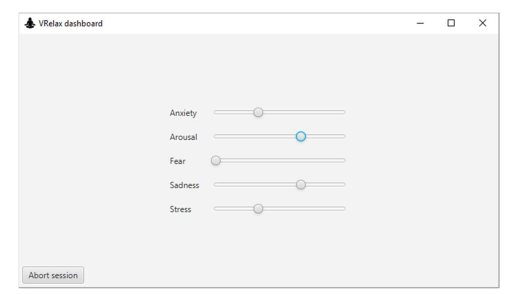

(b)

Fig. 3: VRelax dashboard.

to its demonstrated immersiveness. The erver is a computer with a i7-6700K processor, 16GB memory and a GTX 980 ti Graphics card, running Windows 10. It provides all the modeling functionality both for the user data model and personalization algorithms. These are implemented in Java. In addition, it is also in charge of the rendering of the VR environment, which is then streamed to the HTC Vive. The rendering of the environments has been programmed using Unity (version 2018.3.8) ${ }^{2}$, while the communication between the server and the HMD has been established utilizing the SteamVR library ${ }^{3}$.

At the start of a VRelax relaxation therapy session, the user is presented with a questionnaire on the VRelax dashboard (Figure 3a). Based on the answers, VRelax selects the most suitable environment, renders it and sends it to the HTC Vive, when the patient puts on the HMD. In a real therapy environment, during the session, the user would be monitored through physiological inputs, such as with the sensors within the HTC combined with tracking sensors. Based on the inputs, adaptations on the environment would be triggered. However, in order to showcase the full potential of the proof of concept in a conference (where the stress conditions of the users are

\footnotetext{
${ }^{2}$ https://unity.com/

${ }^{3}$ https://steamcommunity.com/steamvr
}

certainly not the same as in a therapy session), the dashboard offers a manual configuration screen, where different possible stress related feelings can be configured and altered by means of multiple-position sliders (Figure 3b). The stress related feelings have been modeled according to the results shown by Serrano et al. in [10].

With this demonstration, the attendees of the conference will be able to experience first hand the effects of personalizations on VR relaxation techniques.

\section{CONCLUSION}

In this demonstration we present VRelax, a personalized virtual reality approach for relaxation therapy. With a combination of semantic ontologies and self-learning techniques, VRelax models the user preferences and provides an adapted virtual environment. We believe this new type of techniques enables a new area of research for methodologies to improve the experience of patients of relaxation therapy in particular and of people suffering from mental health problems in general.

\section{ACKNOWLEDGMENT}

This research is funded by the imec.icon project PATRONUS, which was co-financed by imec and VLAIO, it brings together the following partners: The Human Link, PreviewLabs, Bazookas, Van Roey Automation. Maria Torres Vega is funded by the Research Foundation Flanders (FWO).

\section{REFERENCES}

[1] L. Valmaggia, L. Latif, M. Kempton, and M. Rus-Calafell, "Virtual reality in the psychological treatment for mental health problems: An systematic review of recent evidence," Psychiatry Res., 2016.

[2] L. B. I. Shah, S. Torres, P. Kannusamy, C. M. L. Chng, H.-G. He, and P. Klainin-Yobas, "Efficacy of the virtual reality-based stress management program on stress-related variables in people with mood disorders: The feasibility study," Archives of Psychiatric Nursing, vol. 29, no. 1, pp. $6-13,2015$.

[3] L. Varvogli and C. Darviri, "Stress management techniques: evidencebased procedures that reduce stress and promote health," Health Science Journal, 2011.

[4] P. M. Lehrer, R. L. Woolfolk, and W. E. Sime, Principles and Practice of Stress Management, Third Edition. Guilford Press, 2007.

[5] S. M. Gerber, M.-M. Jeitziner, P. Wyss, A. Chesham, P. Urwyler, R. M. Müri, S. M. Jakob, and T. Nef, "Visuo-acoustic stimulation that helps you to relax: A virtual reality setup for patients in the intensive care unit," Scientific Reports, no. 7, 2017.

[6] A. Anderson, M. Mayer, A. Fellows, D. Cowan, M. Hegel, and J. Buckey, "Relaxation with Immersive Natural Scenes Presented Using Virtual Reality," Aerospace Medical Human Performance, vol. 88, no. 6, pp. 520-526, june 2017.

[7] Xiao Hang Wang, Da Qing Zhang, Tao Gu, and Hung Keng Pung, "Ontology based context modeling and reasoning using OWL," in IEEE Annual Conference on Pervasive Computing and Communications Workshops, 2004. Proceedings of the Second. Orlando, FL, USA: IEEE, 2004, pp. 18-22.

[8] J. Heyse, F. Ongenae, J. De Letter, A. All, F. De Backere, and F. De Turck, "Design of an ontology for decision support in vr exposure therapy," in Proceedings of Pervasive Health 2019, 2010.

[9] C.-C. Wang, S. R. Kulkarni, and H. V. Poor, "Bandit problems with side observations," IEEE Transactions on Automatic Control, vol. 50, no. 3, pp. 338-355, 2005.

[10] B. Serrano, R. M. Baños, and C. Botella, "Virtual reality and stimulation of touch and smell for inducing relaxation: A randomized controlled trial," Computers in Human Behavior, vol. 55, pp. 1 - 8, 2016. 